Musées, Patrimoine et Culture scientifiques et techniques

$162 \mid 2015$

novembre-décembre 2015

\title{
Numérique : vers un nouvel âge de la médiation culturelle des sciences?
}

\section{Laurent Chicoineau}

\section{OpenEdition \\ Journals}

Édition électronique

URL : http://journals.openedition.org/ocim/1606

DOI : 10.4000/ocim.1606

ISSN : 2108-646X

Éditeur

OCIM

Édition imprimée

Date de publication : 1 novembre 2015

Pagination : 58-62

ISSN : 0994-1908

Référence électronique

Laurent Chicoineau, « Numérique : vers un nouvel âge de la médiation culturelle des sciences? 》, La Lettre de l'OCIM [En ligne], 162 | 2015, mis en ligne le 19 janvier 2016, consulté le 10 décembre 2020. URL : http://journals.openedition.org/ocim/1606 ; DOI : https://doi.org/10.4000/ocim.1606

Ce document a été généré automatiquement le 10 décembre 2020.

Tous droits réservés 


\title{
Numérique : vers un nouvel âge de la médiation culturelle des sciences?
}

\author{
Laurent Chicoineau
}

La plateforme Échosciences développée par La Casemate à Grenoble

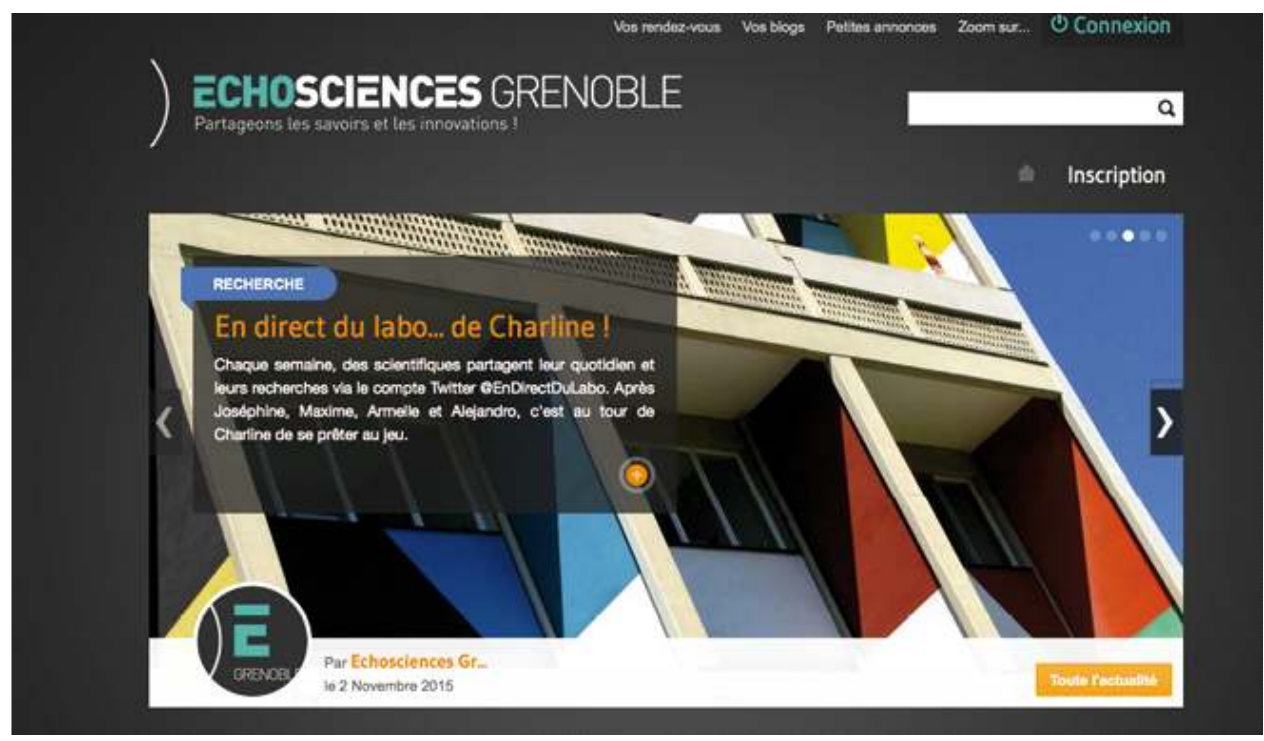

1 Avec le numérique, nous voici directement plongés au cœur des problématiques sociétales, économiques, politiques et éthiques qui accompagnent la diffusion des sciences et des nouvelles technologies dans la société. Le numérique est le terrain par excellence d'observation, de questionnement, et d'expérimentation des diverses formes d'appropriation des sciences et techniques par la société. Il traverse tous les savoirs, toutes les disciplines - on parle même "d'humanités numériques » - offre de nouveaux modes d'apprentissage et de recherche, génère de nouvelles opportunités, de nouveaux emplois ou de nouvelles formes de création de valeur; il n'en questionne pas moins le 
fonctionnement de notre démocratie en bousculant - voire en reconfigurant - nos droits fondamentaux tels que le droit à l'information, à la liberté d'expression, ou à la vie privée. Comme les sciences du vivant ou de la matière, les sciences du numérique nécessitent le développement d'une « recherche et innovation responsables » (responsible research and innovation, notion diffusée sous l'abréviation RRI), pour reprendre l'expression forgée par la Commission Européenne dans la définition de son programme transversal «Sciences avec et pour la société » d'Horizon 2020. Le numérique constitue donc un sujet de choix pour les acteurs de la culture scientifique technique et industrielle (CSTI). Comment s'en emparent-ils? Quelles stratégies numériques développent-ils dans leur mission de médiation culturelle des sciences et des techniques? S'agit-il seulement d'utiliser de nouveaux outils?

D'avril à décembre 2014, nous avons rencontré 162 professionnels issus de diverses structures et familles de la CSTI, dans toute la France ${ }^{1}$. Ils ont bien voulu participer à l'enquête sur leurs pratiques numériques dans le champ de la CSTI ainsi qu'à des tablesrondes organisées dans cinq régions pour explorer collectivement ces pratiques et faire émerger des recommandations. Ces dernières ont fait l'objet d'un rapport de mission, commandité par Geneviève Fioraso, secrétaire d'État à l'Enseignement Supérieur et à la Recherche jusqu'en février 2015, remis fin janvier de la même année ${ }^{2}$. Cette enquête de terrain montre, d'un côté, des médiateurs fortement conscients de l'intérêt et de la nécessité de s'emparer de la thématique "numérique », tant dans ses technologies, ses outils, que dans les enjeux qu'elle soulève. Ceci se traduit, par exemple, par l'intégration en CDI des personnels en charge des communications numériques pour plus de $70 \%$ des structures interrogées, ou bien par le nombre élevé de ces mêmes structures qui ont déjà ou sont en train de réaliser un projet de médiation exploitant des technologies ou outils numériques (83\%). D'un autre côté, ils sont $68 \%$ à mener une réflexion sur leur stratégie numérique tout en estimant, à une très large majorité de l'ensemble des répondants ( 88 \% ) ne pas maîtriser totalement les enjeux. Lorsqu'on leur demande de classer les thèmes prioritaires à leurs yeux (des réseaux sociaux à la data visualisation en passant par la fabrication numérique ou encore l'ouverture et le libre partage des données), tous les thèmes arrivent quasiment à égalité - on observe juste une légère avance pour les thématiques associées aux données.

3 Ainsi, une des premières demandes collectives des professionnels de la CSTI concerne la formation au numérique dans sa dimension communicationnelle et culturelle; au-delà d'une simple maîtrise des outils tels que Twitter ou Facebook par exemple, qu'est-ce que leur mise en œuvre dans un cadre de partage des cultures scientifique technique et industrielle change? Comment articuler médiation humaine et médiation numérique? Les acteurs de la formation professionnelle, comme l'OCIM ou l'AMCSTI, sont déjà mobilisés sur ces enjeux - reste à les démultiplier sur l'ensemble du territoire national et interagir avec d'autres communautés de médiateurs culturels qui font face aux même questionnements et expérimentations (comme le milieu des médiathèques par exemple). 


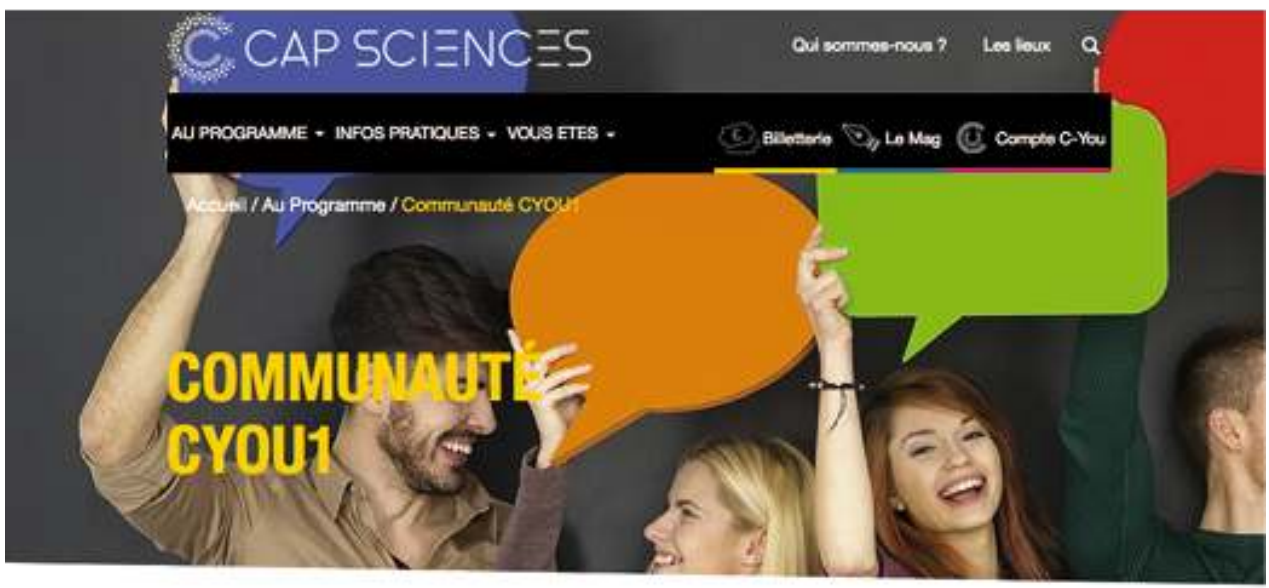

\section{Proposez et participez à des projets collaboratifs !}

Ouverte d 1 1ous, ta communaute croul mssemblo, autour de Cap Sciences, des personnes qui souhaitent s'impliquer et iventuelement proposer des projets

colaboratifs en lien avoc les scimces, les tochniques of I'innovation. Les mernbres de in communaute CYOU1 (actuellement pets de 200) sont thits varies, des etudiants axx retraites, des curreux aux specialistes. Cette cthersite tat toute la rehesse de cente initiative qui cetore la creativite ef rouverture ofesprit des visiteurs dans une ambiance detendue.

\section{Vers une uberisation de la culture numérique?}

C'est un fait, le numérique bouscule les médiateurs. Comme le remarque très justement Bernard Schiele, «[la] cyberculture conduit à l'émergence de nouveaux acteurs et à la marginalisation des acteurs traditionnels de la culture scientifique. Ces nouveaux acteurs proviennent d'horizons divers - du scientifique totalement investi dans son travail à l'amateur passionné -, engagés dans la production et la mise en circulation de nouvelles scientifiques. Ce qui a pour effet de démultiplier les sources et, en conséquence, de défier les façons de faire habituelles. Il y a donc maintenant plus d'acteurs engagés dans la production de nouvelles scientifiques qu'il y a de journalistes ou de communicateurs scientifiques de métier $»^{3}$. On constate en effet que les blogs scientifiques se sont démultipliés sur le Web depuis une dizaine d'années, tout comme les chaînes Youtube thématiques, ou les comptes Twitter éditorialisés par des scientifiques (dont certains sont encore étudiants). Se développe ainsi toute une sphère de médiation des sciences vers la société sans véritable interaction avec les institutions et réseaux traditionnels de CSTI. En matière de partage des cultures scientifique, technique et industrielle, comme dans nombre d'autres secteurs d'activité, le numérique change donc les manières de faire. Peut-on aller jusqu'à évoquer, avec le philosophe Marcel Gauchet, une crise de la médiation? Selon lui, « Internet est le média absolu, la médiation qui supprime toutes les autres médiations, qui les rend inutiles $»^{4}$ et le domaine de la CSTI ne semble pas échapper à la désintermédiation qui touche de nombreux domaines dont le journalisme, l'édition ou l'éducation. Va-t-on vers une « uberisation $»^{5}$ de la culture scientifique? 
Déjà, en 2007, Dominique Boullier, directeur du Laboratoire des usages en technologies de l'information numérique (LUTIN), et son équipe, publiaient une étude sur les stratégies éditoriales des acteurs de CSTI sur le Web qui pouvait laisser entrevoir le phénomène ${ }^{6}$. D'après lui, la logique d'équipement dominait, ce qu'il baptisait «l'effet vitrine », dont l'unique objectif était de faire venir des visiteurs dans les institutions. Il regrettait le manque d'animations interactives en ligne - alors que bien souvent les acteurs culturels scientifiques développent de nombreux interactifs dans leurs expositions - et remarquait l'absence de liens avec les amateurs de science pourtant très actifs de leur côté grâce aux possibilités offertes par le Web 2.0. «Malgré le caractère quasi évident d'une offre Web en provenance de ce champ, il n'est pas certain que, malgré les bonnes intentions et les recherches d'innovation, l'offre des institutions de diffusion de la CSTI sur le Web soit adaptée aussi bien aux publics déjà conquis comme aux publics à conquérir ", soulignaitil. Et de conclure sur quelques pistes qui pourraient structurer une nouvelle stratégie de partage des CSTI sur le Web, comme par exemple passer « De la CSTI officielle à la CSTI débat », ou encore " De la CSTI événement à la CSTI capitalisante » en s'appuyant sur « de véritables communautés qui pourraient prendre en charge certains des domaines à partir d'un événement donné pour le faire vivre au-delà du moment même d'animation publique et conserver ses traces vivantes $»$.

De telles perspectives ont été ou sont en cours de mise en œuvre, ici et là en France, et commencent à se démultiplier. On peut citer les expérimentations conduites depuis 2012 par les six centres de science du programme Inmédiats ${ }^{7}$ autour des réseaux sociaux thématiques territoriaux (comme la plateforme Échosciences ou la communauté CYou1), la transformation numérique des Exposciences, avec la mise en œuvre d'une plateforme Web d'accompagnement et de valorisation des projets portés par des jeunes, ou encore les projets de science participative, tel Vigie Nature, porté par le Muséum national d'Histoire naturelle. Car le numérique permet de renouer avec cette pratique ancienne de contribution des citoyens à la recherche scientifique. Par connexion directe sur le Web, des chercheurs peuvent compter directement sur la mobilisation d'un grand nombre de personnes à travers des programmes de science participative. Souvent gamifiés (i.e. mis en scène selon les règles du jeu vidéo), ces programmes visent à mobiliser des individus amateurs, souvent passionnés, pour réaliser des tâches de dénombrement, repérage, observation à partir d'images scientifiques (Galaxy Zoo, par exemple) ou in situ (Vigie Nature). 
PROTO 204, une plateforme de partage facilitatrice de mise en relation

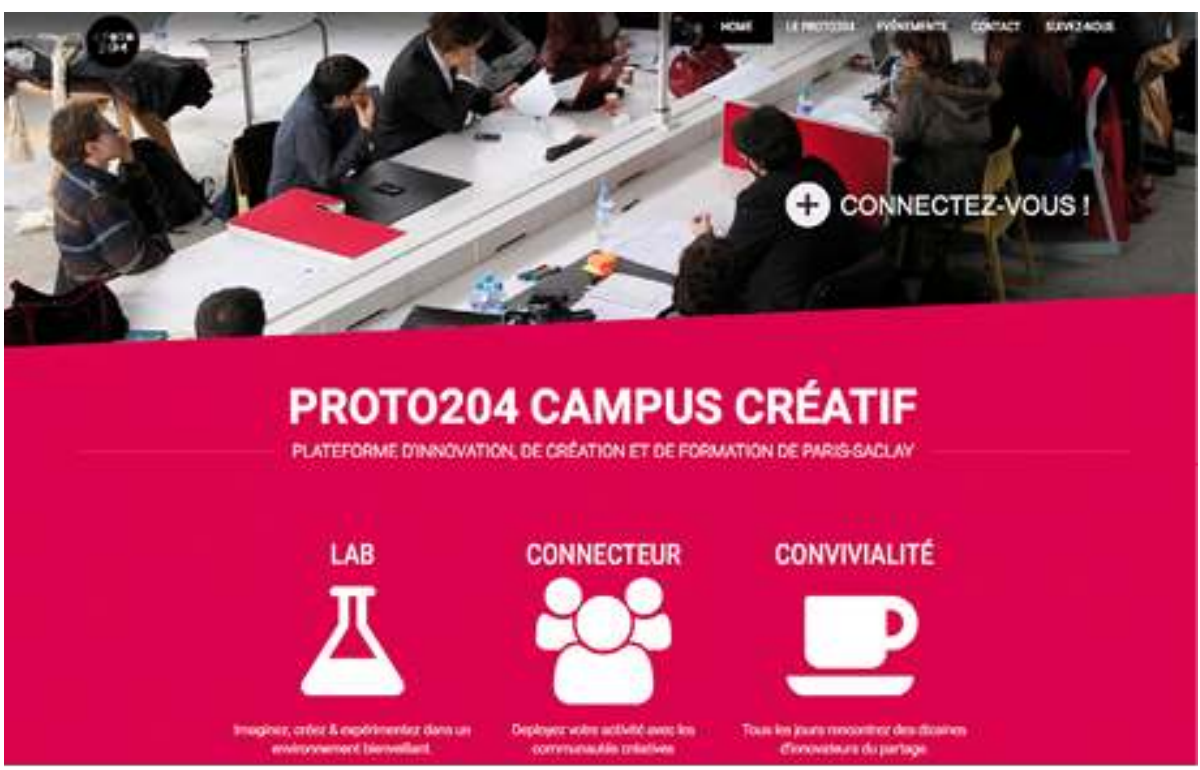

\section{Menaces et opportunités}

7 La transition numérique amène les acteurs de la médiation culturelle des sciences à repenser et faire évoluer non seulement leurs pratiques, mais aussi leur positionnement dans le champ social et culturel. Il nous semble, avec Bernard Schiele, que « la donne a changé. Il ne s'agit plus seulement d'inviter les médiateurs à rechercher les meilleures formes de communication, ni d'engager les scientifiques à redoubler d'efforts pour devenir de meilleurs communicateurs, il faut (...) que médiateurs, scientifiques et acteurs sociaux s'engagent les uns avec les autres pour, collectivement, aborder les problèmes et les enjeux qui nous confrontent tous $»^{8}$. Cette prise de conscience se diffuse auprès d'un nombre croissant de médiateurs et de scientifiques, mais elle n'a pas encore atteint toutes les couches du corps social, et notamment les décideurs. Côté médiateurs, on observe de nouvelles pratiques et surtout de nouveaux positionnements sur le terrain, moins focalisés sur la vulgarisation des sciences que sur la mise en relation, en réseau, de personnes et de communautés. Certains vont même jusqu'à proposer de transformer le terme " médiateur » en " connecteur ", argumentant sur les vertus du décloisonnement tant pour l'impact des actions de ce genre sur les publics que pour la montée en compétences et en capacité des praticiens et acteurs de la médiation impliqués - ce que les anglo-saxons nomment empowerment. Cette dynamique est portée, entre autres exemples, par Ronan James, passé par les associations TRACES, Atomes crochus et Doc en stock, après une thèse en climatologie à l'ENS et l'UPMC. Actuellement animateur de l'espace PROTO 204 sur le plateau de Saclay, il envisage son travail de médiateur plutôt comme un facilitateur de mise en relation, voire un détecteur de tendances dont la priorité est d'accompagner et d'accélérer le développement de projets à travers la mobilisation "d'agents d'ouverture ", c'est-à-dire d'individus qui permettent d'ouvrir le système là où ils sont, et ainsi le décloisonner. Lieu physique implanté sur un territoire à forte densité universitaire et scientifique, il est frappant de constater combien le PROTO 
204 fonctionne sur le modèle d'une plateforme Web de partage et de mise en relation. " Seul, je n'ai pas les moyens de mettre en œuvre une programmation culturelle au sens traditionnelle $\mathrm{du}$ terme, explique Ronan James. Aussi nous fonctionnons en programmation ouverte, c'est-à-dire que toutes les actions sont construites par et avec les communautés du plateau de Saclay et au-delà. C'est un projet en mode crowdsourcing, fonctionnant essentiellement par événements, avec en plus des ateliers et master-class pour apprendre à coder ou pitcher son projet $"^{9}$. On retrouve la même dynamique et vision de la médiation dans la Maison de la Recherche et de l'Imagination (MRI) conçue et développée par Relais d'science à Caen, ou encore dans le projet Le Catalyseur de l'université Paul Sabatier à Toulouse, en cours de développement en partenariat avec Science Animation Midi Pyrénées.

8 Côté décideurs, cette nouvelle approche de la médiation semble plus lointaine. Faut-il y voir un des nombreux symptômes de la déconnexion des élites décrite par la journaliste Laure Belot dans sa passionnante enquête sur " Comment Internet change l'ordre établi » ${ }^{10}$ ? Force est de constater que pour de nombreux établissements culturels sous pilotage de collectivités territoriales (mais l'État ne fait pas mieux en la matière), la transition numérique et ses implications vers une "nouvelle médiation» n'est pas toujours accompagnée avec la vigueur nécessaire. D'abord tout simplement en matière d'équipement et d'infrastructure, car les services informatiques des communes ou des départements n'ont pas, à quelques exceptions près, réellement anticipé les nouveaux usages et donc les nouveaux besoins exprimés par les équipes des musées et centres culturels scientifiques (il suffit d'enquêter sur les accès WIFI pour le public dans les musées par exemple). Ensuite - et peut-être surtout - en matière de décision, d'allocation de ressources et d'arbitrage. Un enjeu majeur apparaît alors, celui de la formation de décideurs. Il s'agit de leur faire prendre conscience des potentialités des outils et des pratiques numériques pour le partage des savoirs et le développement des territoires. L'idéal serait d'accomplir cette tâche par l'expérience, car la formation au numérique ne peut pas se réaliser uniquement de manière théorique, par la lecture d'articles ou la participation à des conférences spécialisées; elle nécessite le développement d'une pratique personnelle, visant l'appropriation par l'usage en conditions réelles. Pourquoi ne pas imaginer, avec Yves-Armel Martin, directeur du Living Lab Érasme, la création d'un « SPOC $»^{11}$ à destination des managers et décideurs qui viserait à les faire plonger directement dans les usages numériques? 
Muséomix, une occasion pour le musée de démystifier l'appropriation du numérique dans la médiation culturelle et patrimoniale.

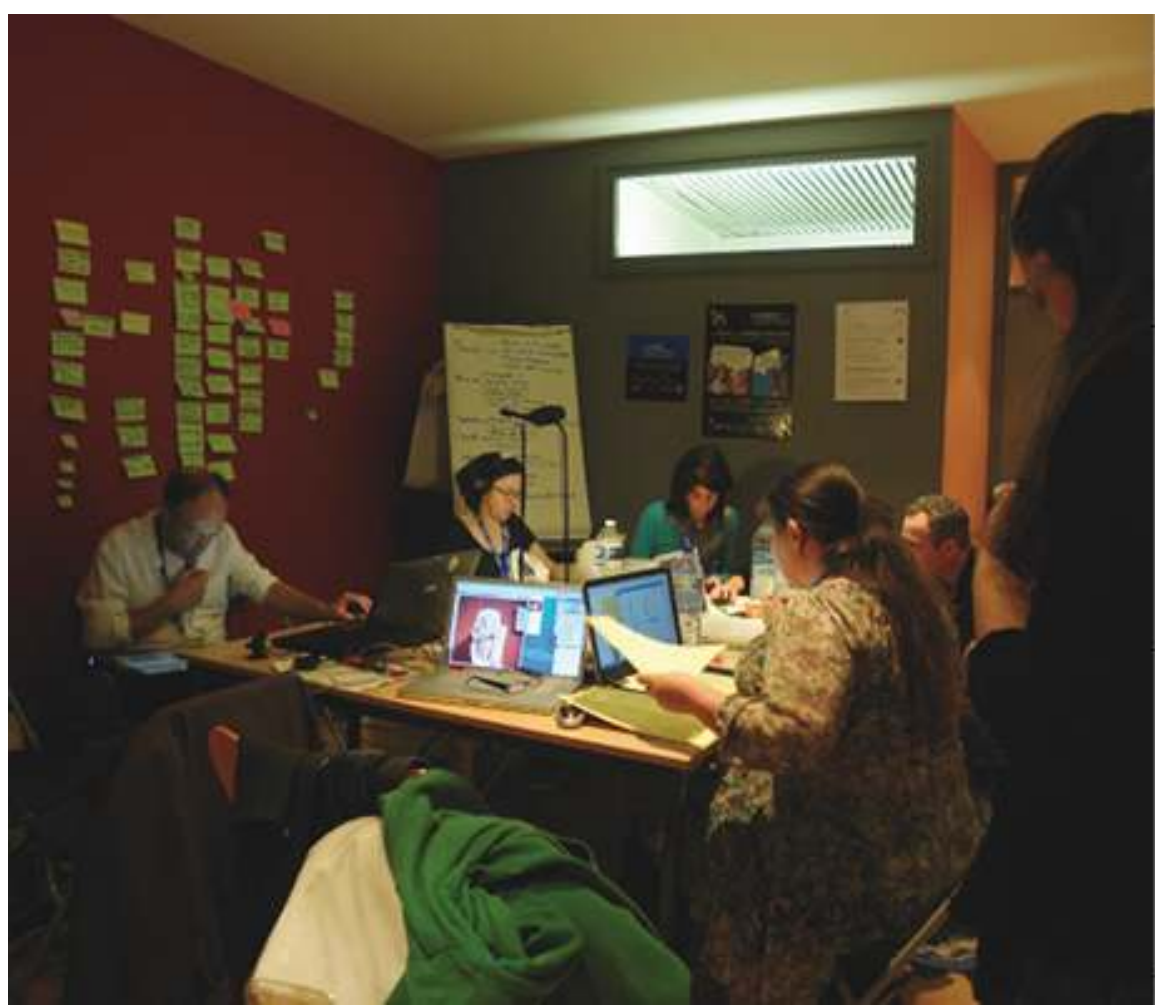

(c) OCIM/Sylvie Grange

\section{De l'importance des espaces physiques de médiation}

L'objectif de formation aux pratiques numériques par l'usage trouve une excellente illustration dans l'expérience Museomix. Imaginée sur l'idée de remixer le musée par un collectif d'acteurs constitué de professionnels des musées, de la communication et des nouvelles technologies, Museomix croise culture numérique et patrimoine, en invitant des professionnels ou amateurs de la médiation, du design, de la programmation informatique, de la scénographie à créer, par équipes pluridisciplinaires et en temps limité ( 3 jours), des dispositifs de médiation directement au sein du musée. L'opération propose une immersion intense dans les nouvelles formes de médiation culturelle numérique de façon collective, concrète (via le principe de prototypage rapide) et réflexive (des experts sont invités à suivre et accompagner le processus et témoigner à la fin dans des debriefings collectifs). D'après les responsables des musées, qui postulent chaque année en plus grand nombre pour accueillir un Museomix, comme pour leurs équipes, cette opération contribue à démystifier l'appropriation du numérique dans la médiation culturelle et patrimoniale en montrant les processus de conception et de production, un peu comme une formation continue en accéléré. Ainsi, alors que beaucoup voient dans le numérique la question (voire le risque) de la dématérialisation, de la disparition des objets et du délitement du lien social, notre travail au plus près des acteurs a révélé, au contraire, la nécessité de maintenir et développer des lieux d'échange, de pratiques collectives, des espaces de rencontres à la fois événementielles et régulières. La transition numérique est une chance pour les institutions culturelles, 
muséales ou non, de (re)jouer un rôle déterminant dans l'accompagnement et l'amélioration de notre « vivre ensemble ». Parce qu'elles sont investies par le public d'un degré de confiance encore élevé ${ }^{12}$, parce qu'elles peuvent mettre en œuvre les conditions d'émergence des initiatives d'un territoire et de connexion entre les domaines d'activité, parce qu'elles visent sans cesse à décloisonner, abattre les frontières entre les disciplines et les acteurs, les institutions culturelles scientifiques sont l'un des moteurs de l'innovation sociale dans une société dite « de la connaissance ».

Partager les cultures scientifique, technique et industrielle à l'ère numérique ne se résume alors pas à l'apprentissage du code informatique par les médiateurs mais suppose de s'en approprier les usages, les enjeux et les pratiques. Ceci implique une réflexion sur la médiation scientifique en tant que telle qui nécessite à la fois d'accompagner les acteurs historiques au changement et d'élargir le champ à de nouveaux acteurs et pratiques, dans un esprit de fertilisation croisée. «Il ne suffit plus de savoir lire et de savoir écrire, il nous faut maintenant d'autres savoirs et d'autres pédagogies. Des savoirs issus du numérique, de ses critères émergents et de ses repères propres ${ }^{13}$.

\section{NOTES}

1. Services médiation ou communication des universités et centres de recherche, enseignants du primaire et du secondaire, enseignants-chercheurs, associations d'éducation populaire, musées et muséums d'Histoire naturelle, centres de science (CCSTI) et collectivités territoriales.

2. Ce rapport, non publié pour l'instant, est à l'étude dans les services de Thierry Mandon, nouveau secrétaire d'État à l'Enseignement Supérieur et la Recherche depuis juin 2015.

3. Schiele, B. Médiation des sciences : cinq choses à garder en tête, Découvrir, publication en ligne de l'Association francophone pour le savoir (AFCAS), mars 2015. www.acfas.ca/publications/ decouvrir/2015/03/mediation-sciences-cinq-choses-garder-en-tete

4. Intervention de Marcel Gauchet le 22 février 2007 au Colloque Public sur l'avenir du Livre organisé par le Centre National du Livre. Texte disponible en ligne : http:// gauchet.blogspot.fr/2007/04/les-crises-de-la-mdiation.html

5. En référence à la société UBER et sa stratégie de concurrence des taxis en permettant à n'importe quel conducteur, via une application mobile, de transformer son véhicule en taxi. Voir www.lemonde.fr/les-decodeurs/article/2015/06/26/de-quoi-l-uberisation-est-elle-lenom_4662261_4355770.html

6. Boullier, D. Web CSTI : Le Web est un autre monde ! La Lettre de l'OCIM, nº117, mai-juin 2008, pp. 4-13.

7. Inmédiats (Innovation, Médiation, Territoires) est un programme financé par les Investissements d'avenir suite à l'appel à projet "Culture scientifique - égalité des chances". Il regroupe les centres de science de Bordeaux (Cap Sciences), Toulouse (Science Animation), Grenoble (La Casemate), Rennes (Espace des sciences), Caen (Relais d'science) et Paris (Universcience). Voir www.inmediats.fr

8. Op. cit.

9. Entretien réalisé le 19/11/2014 par l'auteur. 
10. Belot, L. La déconnexion des élites, comment internet change l'ordre établi. Paris : Les Arènes, 2015. http://ladeconnexiondeselites.fr/

11. Small Private Online Course. Cours en ligne destiné à un petit groupe privé. Le concept se définit comme une évolution hybride des MOOC par les milieux académiques. Voir Wikipédia : https:// fr.wikipedia.org/wiki/SPOC

12. Le Marec, J. Publics et musées, la confiance éprouvée. Paris : L'Harmattan, 2007

13. Doueihi, M. Qu'est-ce que le numérique? Paris : PUF, 2013.

\section{RÉSUMÉS}

L'auteur, rédacteur d'un rapport sur les pratiques numériques dans le champ de la CSTI, dresse un panorama de ces pratiques, qui, en pleine évolution, apparaissent moins orientées aujourd'hui vers la vulgarisation des sciences que vers la mise en réseau de personnes et de communautés et permettent ainsi de renouer avec cette pratique ancienne de contribution des citoyens à la recherche scientifique.

\section{INDEX}

Mots-clés : médiation culturelle, sciences, numérique

\section{AUTEUR}

\section{LAURENT CHICOINEAU}

directeur de La Casemate, CCSTI de Grenoble

laurent.chicoineau@lacasemate.fr 